\title{
Inhibition of miR-34a prevents endothelial cell apoptosis by directly targeting HDAC1 in the setting of atherosclerosis
}

\author{
YANGWEI LI ${ }^{1,2}$, KANG ZHANG ${ }^{2}$ and WEI MAO ${ }^{1}$ \\ ${ }^{1}$ Department of Cardiovascular Medicine, The First Clinical College of Zhejiang Chinese Medical University, Hangzhou, \\ Zhejiang 310006; ${ }^{2}$ Department of Cardiovascular Medicine, Dongyang People's Hospital, Jinhua, Zhejiang 322103, P.R. China
}

Received September 10, 2017; Accepted December 21, 2017

DOI: $10.3892 / \mathrm{mmr} .2018 .8411$

\begin{abstract}
Despite recent medical advances, atherosclerosis is a global burden accounting for numerous mortalities and hospital admissions. MicroRNAs (miRNAs/miRs) regulate cardiovascular biology and disease, but the role of microRNA-34a in atherosclerosis remains unclear. In the present study, it was demonstrated that miR-34a was highly expressed in atherosclerotic lesions and oxidized low-density lipoprotein (Ox-LDL)-treated human aortic endothelial cells (HAECs) (atherosclerotic cell model) using reverse transcription-quantitative polymerase chain reaction. The expression of histone deacetylase (HDAC) 1 was reduced in atherosclerotic lesions and Ox-LDL treated HAECs. TargetScan predicted that HDAC1 is the potential target of miR-34a and the double-luciferase reporter assay confirmed that HDAC1 was directly targeted by miR-34a. Furthermore, miR-34a inhibitor significantly enhanced the cell viability of HAECs and the cell apoptosis was suppressed. In addition, the expression of apoptotic-related proteins was detected by western blotting. The results showed that miR-34a inhibitor significantly upregulated B-cell lymphoma 2, procaspase-3, procaspase- 9 and proto-oncogene c-Myc protein expression, and downregulated the expression of $\mathrm{p} 21$. In contrast, co-transfection of HDAC1-small interfering RNA and miR-34a inhibitor eliminated the effects of miR-34a on HAECs. This indicated that miR-34a inhibitor promoted cell viability and prevented cell apoptosis of HAECs through regulating $\mathrm{HDAC} 1$. In conclusion, it was demonstrated that miR-34a promoted atherosclerotic formation by modulating the proliferation and apoptosis of HAECs, and regulating the expression of apoptosis-related proteins by targeting HDAC1.
\end{abstract}

Correspondence to: Dr Wei Mao, Department of Cardiovascular Medicine, The First Clinical College of Zhejiang Chinese Medical University, 54 Youdian Road, Hangzhou, Zhejiang 310006, P.R. China E-mail: maowei1801@163.com

Key words: atherosclerosis, miR-34a, HDAC1, HAECs, apoptosis

\section{Introduction}

Atherosclerosis is a chronic inflammatory disease that causes vascular walls that can cause cardiovascular disease such as stroke, myocardial infarction, and peripheral blood artery disease $(1,2)$. In developed countries, atherosclerosis is a leading cause of mortality. It remains a huge challenge to solve this global clinical problem. The pathogenesis of atherosclerotic lesion formation is a multistage process. Inflammation is a major component of atherosclerosis and considered to play a role in all developmental stages of the diseases $(3,4)$. So far, few have known that the complex upstream gene regulators involved in response to atherosclerosis.

MicroRNAs are evolutionary conserved non-coding RNAs of about 19-25 nucleotides, function by regulating one or more mRNA to regulate gene expression for translation inhibition or cleavage $(5,6)$. With regard to miRNA function, they play a key role in cell proliferation, cell death and organ development $(7,8)$. Recent studies have revealed that miRNAs play a key role in the pathophysiological processes of atherosclerosis. MicroRNAs control the senescence and dysfunction of endothelial cells, proliferation and migration of vascular smooth muscle cells, and macrophage-driven cytokine production and polarization.

MicroRNA-34a (miR-34a) is located in the region of chromosome 1p36.23, and usually, it is expressed aberrantly in multiple types of diseases such as human tumors (9), atherosclerotic cardiovascular diseases $(10,11)$, and other types of diseases. MiR-34a is suggested highly expressed in atherosclerosis patients and played critical roles in the regulation of various cell biological events including cell proliferation, differentiation, apoptosis, etc. Endothelial cell (EC) apoptosis is a crucial process for the development of atherosclerosis.

To the best of our knowledge, no precise studies have been made about the role of miR-34a in cell apoptosis in atherosclerosis progress. This study we will investigate the exact role of miR-34a in cell apoptosis in atherosclerosis.

\section{Materials and methods}

Tissue samples. A total of 15 pairs of atherosclerotic lesion tissues and normal veins were obtained during treatment from 15 patients at Dongyang People's Hospital. All tissues were immediately stored in liquid nitrogen until use. All the protocols were approved by the Ethics Committee of 
Dongyang People's Hospital. Informed consent was obtained from each patient.

Cell culture. The human aortic endothelial cells (HAECs) were cultured in RPMI-1640 medium supplemented with $10 \%$ fetal bovine serum (Gibco; Thermo Fisher Scientific, Inc., Waltham, MA, USA) and $1 \%$ penicillin/streptomycin. All cells were incubated in a humidified incubator at $37^{\circ} \mathrm{C}$ and $5 \%$ $\mathrm{CO}_{2}$

Establishment of atherosclerotic cell model. Ox-LDL was used to establish the atherosclerotic cell model. Native LDL (Sigma-Aldrich; Merck KGaA, Darmstadt, Germany) was oxidized by exposure to $\mathrm{CuSO} 4(5 \mu \mathrm{mol} / 1$ free $\mathrm{Cu} 2+$ concentration) in PBS at $37^{\circ} \mathrm{C}$ for $24 \mathrm{~h}$. HAECs were treated with $50 \mu \mathrm{g} / \mathrm{ml}$ ox-LDL for $24 \mathrm{~h}$.

Cell transfection. The miR-34a mimic (50 nM), miR-34a inhibitor $(50 \mathrm{nM})$, the negative control $(50 \mathrm{nM})$ (Shanghai GenePharma Co., Ltd., Shanghai, China), $1 \mu \mathrm{g}$ Control-siRNA (empty vector), $1 \mu \mathrm{g}$ HDAC1-siRNA (Santa Cruz Biotechnology, Inc., Dallas, TX, USA) or $50 \mathrm{nM}$ miR-34a inhibitor+1 $\mu \mathrm{g}$ HDAC1-siRNA, was transfected into HAECs respectively using $30 \mu \mathrm{l}$ Lipofectamine 2000 (Invitrogen; Thermo Fisher Scientific, Inc., Waltham, MA, USA) according to the manufacturer's instructions. Transfected cells were incubated at $37^{\circ} \mathrm{C}$ in an atmosphere of $5 \% \mathrm{CO}_{2}$ for $48 \mathrm{~h}$.

RNA extraction and reverse transcription-quantitative $P C R$. Total RNA of atherosclerotic tissues or HAECs was extracted by using TRIzol reagent (Invitrogen; Thermo Fisher Scientific, Inc.), and cDNAs were synthesized using miScript Reverse Transcription kit (Qiagen GmbH, Hilden, Germany) according to the manufacturer's instructions. The primers for reverse transcription and amplification of miR-34a, HDAC1 and U6 were designed and synthesized by GenScript Co. Ltd., (Nanjing, China). Quantitative real-time PCR was conducted to detect miR-34a and HDAC1 mRNA using the SYBR Premix Ex Taq ${ }^{\mathrm{TM}}$ II (TliRNaseH Plus) kit (Takara Biotechnology Co., Ltd., Dalian, China) using the Bio-Rad Laboratories, Inc., (Hercules, CA, USA) machine, with the U6 small nuclear RNA and GAPDH as internal normalized references, respectively. The primer sequences for qPCR were as follows:U6-Forward: 5'AAAGCAAATCATCGG ACGACC3'; U6-Reverse: 5'GTACAACACATTGTTTCC TCGGA3'; GAPDH-Forward: 5'GAAGGTGAAGGTCGG AGTC3'; GAPDH-Reverse: 5'GAAGATGGTGATGGGATT TC3'; miR-34a-Forward: 5'CAGCCTGGAGGAGGATCG A3'; miR34a-Reverse: 5'TCCCAAAGCCCCCAATCT3'; HDAC1-Forward: 5'CTACTACGACGGGGATGTTGG3'; HDAC1-Reverse: 5'GAGTCATGCGGATTCGGTGAG3'. The $2^{-\triangle \Delta \mathrm{Cq}}$ method was applied to quantify the relative gene expressions (12).

Cell proliferation assay. Cell Count Kit-8 assay (CCK-8; Dojindo Molecular Technologies, Inc., Kumamoto, Japan) was used as a qualitative marker for cell viability. After $48 \mathrm{~h}$ transfection with miR-34a mimic, miR-34a inhibitor, the negative control or miR-34a inhibitor+ HDAC1-siRNA, HAECs were seeded into 96 well plates in triplicate at $5 \times 10^{3}$ cells per well. At $0,24,48$, and $72 \mathrm{~h}, 10 \mu \mathrm{l}$ of CCK-8
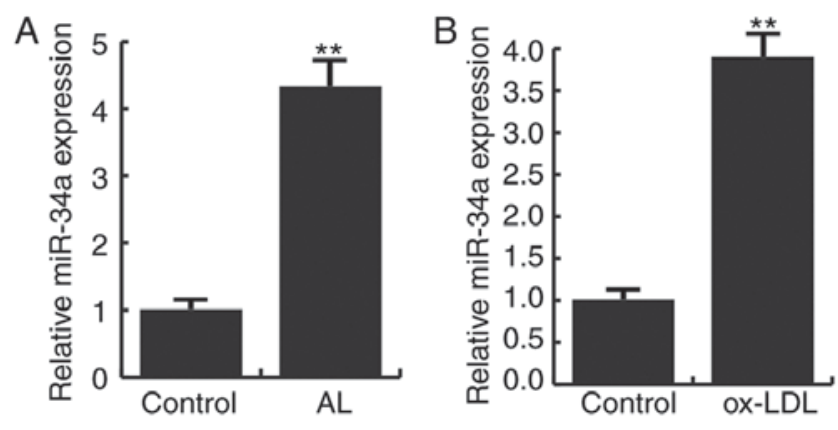

Figure 1. Expression of miR-34a in atherosclerosis. The expression level of miR-34a was detected by qRT-PCR. (A) The relative miR-34a expression in normal veins (Control) and atherosclerotic lesion tissues (AL); (B) the relative miR-34a expression without ox-LDL (Control) and HAECs treated with ox-LDL (ox-LDL). ${ }^{* *} \mathrm{P}<0.01$ vs. Control.

solution mixed with $90 \mu \mathrm{l}$ of RPMI-1640 was added to each well. And after $2 \mathrm{~h}$ of incubation, the absorbance was measured at $570 \mathrm{~nm}$.

Apoptosis assay. HAECs were transfected with miR-34a mimic, miR-34a inhibitor, the negative control or miR-34a inhibitor+ HDAC1-siRNA, and $48 \mathrm{~h}$ after transfection, the cells then were subjected to apoptosis assay. Then 106 treated cells were stained with Annexin V and PI using an apoptosis detection kit (BD Biosciences, Franklin Lakes, NJ, USA). According to the manufacturer's instructions, after incubation for $15 \mathrm{~min}$ in the dark, cell apoptosis were then detected by flow cytometry.

Luciferase reporter assay. For confirmation of direct target binding, the wild type and mutant 3'UTR of HDAC1 identified by TargetScan was cloned into a pmiR-RB-ReportTM dual luciferase reporter gene plasmid vector (Guangzhou RiboBio Co., Ltd., Guangzhou, China). The UTR region of candidate target gene was inserted downstream of the sequence of renilla luciferase, which is designed for reporter fluorescence. For luciferase reporter analysis, HAECs were co-transfected with luciferase reporter vectors and control mimic or miR-34a mimic using Lipofectamine 2000. After 48 h, luciferase activity was analyzed by the dual-luciferase assay system (Promega Corporation, Madison, WI, USA) according to the manufacturer's protocols.

Western blot analysis. HAECs were transfected with miR-34a mimic, miR-34a inhibitor, the negative control or miR-34a inhibitor+ HDAC1-siRNA for $48 \mathrm{~h}$, then cells were collected and total proteins were extracted in $40 \mathrm{mM}$ Tris- $\mathrm{HCl}(\mathrm{pH} 7.4)$ containing $150 \mathrm{mM} \mathrm{NaCl}$ and $1 \%(\mathrm{v} / \mathrm{v})$ Triton X-100, supplemented with protease inhibitors. Protein concentration was determined using the bicinchoninic acid protein assay (Pierce; Thermo Fisher Scientific, Inc.). Equal amounts of protein were resolved on 10\% SDS-PAGE gels, and then transferred to a PVDF membrane (EMD Millipore, Billerica, MA, USA). After blocking with 5\% skimmed milk in TBST, then probed with antibodies against Bcl-2 (cat no. 4223; dilution: 1:1,000), procaspase-3 (cat no. 9664; dilution: 1:1,000), procaspase-9 (cat no. 9501; dilution: 1:1,000), c-Myc (cat no. 13,987; dilution: 1:1,000); p21 (cat no. 2947; dilution: 1:1,000); $\beta$-actin (cat no. 4970; dilution: 1:1,000) 
A

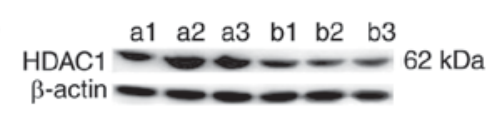

C

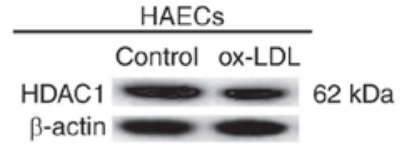

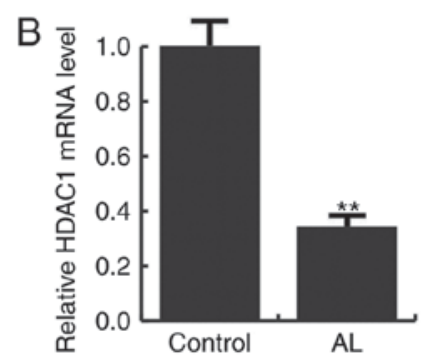
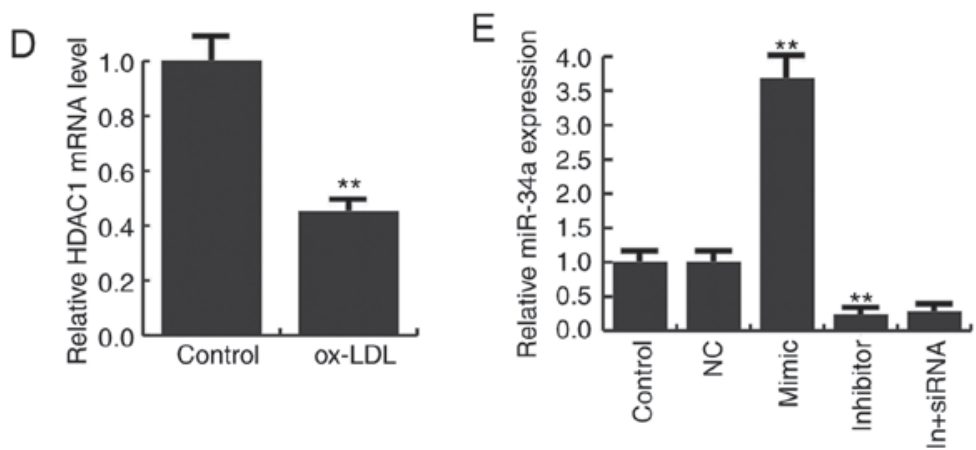

Figure 2. Expression of HDAC1 in atherosclerosis. (A) Protein level of HDAC1 in normal veins (a1-a3) and atherosclerotic lesion tissues (b1-b3); (B) the relative HDAC1 mRNA expression in atherosclerotic lesion tissues (AL) and normal veins (Control); (C) protein level of HDAC1 in $\mathrm{HAECs}$ treated with (ox-LDL) or without (Control) ox-LDL; (D) the relative mRNA expression of HDAC1 in HAECs treated with (ox-LDL) or without (Control) ox-LDL; (E) the relative miR-34a expression in HAECs in different groups, Control: cells without any treatment, ox-LDL: cells treated with ox-LDL, NC: cells transfected with the negative control, mimic: cells transfected with miR-34a mimic, inhibitor: cells transfected with miR-34a inhibitor, In+siRNA: cells co-transfected with miR-34a inhibitor and HDAC1-siRNA. ${ }^{* *} \mathrm{P}<0.01 \mathrm{vs}$. Control.

(all from Cell Signaling Technology, Inc., Danvers, MA, USA), membranes were subsequently incubated with horseradish peroxidase (HRP) conjugated secondary antibody (Anti-rabbit IgG, HRP-linked Antibody; cat no. 7074; dilution: 1:5,000). Immunoreactive bands were visualized using the enhanced chemiluminescence detection system. The protein levels of the stripes were normalized based on the gray value of $\beta$-actin.

Statistical analysis. SPSS v17.0 software was used to analyze the data. Values are expressed as mean \pm SD of experiments performed in triplicate. Data were analyzed by one-way ANOVA or Student's t-test. $\mathrm{P}<0.05$ was considered to indicate a statistically significant difference.

\section{Results}

MiR-34a is highly expressed in atherosclerosis. Fifteen pairs of atherosclerotic lesion tissues and normal veins were recruited in this study. Q-RT PCR result showed that compared to the normal veins, the expression of miR-34a were significantly increased in atherosclerotic lesion tissues (Fig. 1A). In addition, Ox-LDL induction significantly increased the expression of miR-34a in HAECs (Fig. 1B). These data suggest that miR-34a is highly expressed in atherosclerosis.

HDACl is low expressed in atherosclerosis. We next studied the expression of HDAC1 in atherosclerosis, and we found that the expression of HDAC1 was reduced both in atherosclerotic lesions and Ox-LDL treated HAECs (Fig. 2A-D).

For further study the role of miR-34a in atherosclerosis, miR-34a mimic, miR-34a inhibitor, the negative control (NC), Control-siRNA (empty vector), HDAC1-siRNA, or miR-34a inhibitor+HDAC1-siRNA was transfected into HAECs respectively, the transfection efficiency was detected by qRT-PCR and western blotting (Figs. 2E and 3).
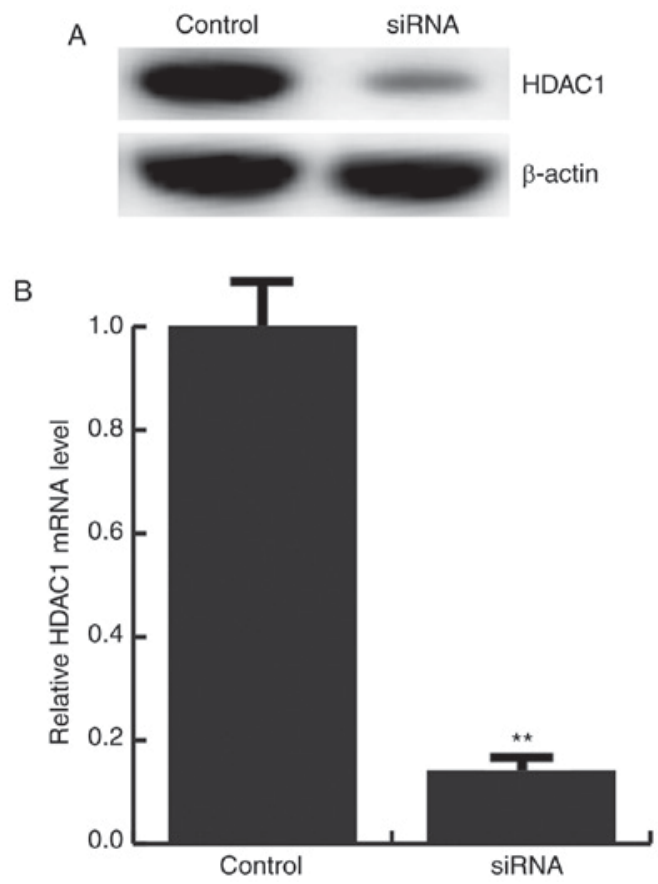

Figure 3. Expression of HDAC1 in HAECs transfected with HDAC1-siRNA. (A) Protein level of HDAC1 in HAECs transfected with HDAC1-siRNA or Control-siRNA; (B) the relative HDAC1 mRNA expression in HAECs transfected with HDAC1-siRNA (siRNA) or Control-siRNA (Control). ${ }^{* *} \mathrm{P}<0.01$ vs. Control.

HDAC1 is the target gene of miR-34a. We used TargetScan to predict the potential targets of miR-34a, HDAC1 was identified as a potential miR-34a target gene (Fig. 4A). And the luciferase reporter assay showed that the luciferase activity was significantly reduced in the HAECs co-transfected of miR-34a with HDAC1-UTR-WT reporter plasmids, but co-transfection of miR-34a with HDAC1-UTR-MUT reporter plasmids did not (Fig. 4B). To further confirm that miR-34a regulates HDAC1 expression in HAECs, miR-34a mimic, 
C

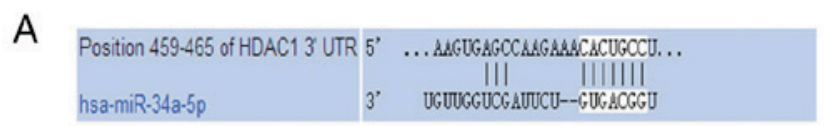

B

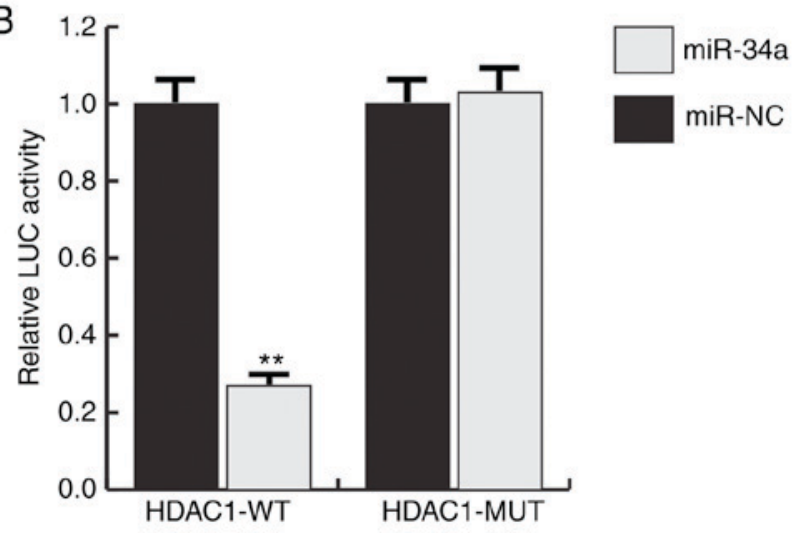

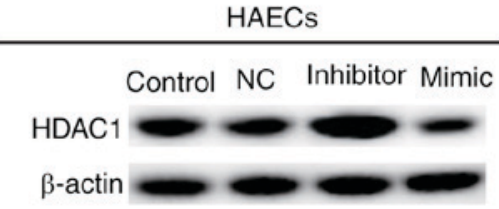

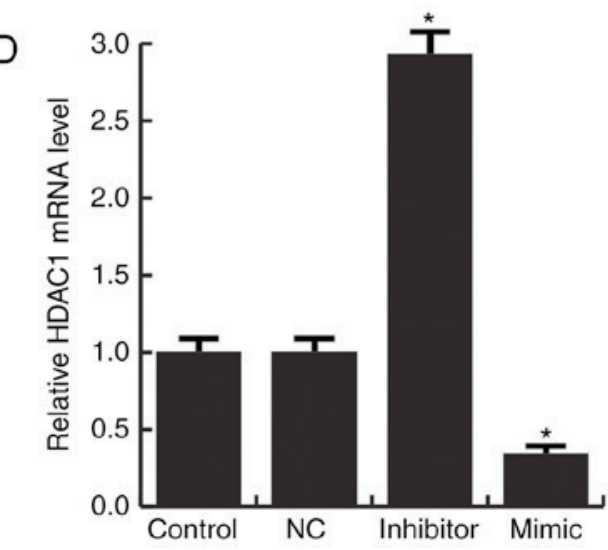

Figure 4. HDAC1 is a direct target of miR-34a. (A) Interaction between miR-34a and 3'UTR of HDAC1 was predicted using TargetScan target site prediction software; (B) Luciferase activity of a reporter containing a wild-type HDAC1 3'UTR or a mutant HDAC1 3'UTR are presented. 'HDAC1-3'UTR-MUT' indicates the HDAC1 3'UTR with a mutation in the miR-34a binding site. UTR, untranslated region. $48 \mathrm{~h}$ after cell transfection, protein (C) and mRNA (D) levels of HDAC1 in HEACs in different groups were determined by using western blotting and qRT-PCR respectively. All data are presented as the mean \pm SD of three independent experiments. ${ }^{*} \mathrm{P}<0.05,{ }^{* *} \mathrm{P}<0.01$ vs.control.

miR-34a inhibitor and negative control (NC) were transfected into HAECs. The results showed that miR-34a mimic significantly inhibited the expression of HDAC1, and miR-34a inhibitor significantly enhanced the expression of HDAC1 (Fig. 4C and D). Together, these data indicate that miR-34a directly target HDAC1.

MiR-34a inhibitor enhances the cell viability of HAECs. Given our limited understanding of the role played by miR-34a in HAECs, we examined the effects of miR-34a gain and loss-of-function on atherosclerosis. The CCK-8 results showed that Ox-LDL induction significantly reduced the viability of HAECs, and when compared with ox-LDL treatment alone, miR-34a inhibitor significantly enhanced the cell viability of HAECs, whereas HDAC1-siRNA eliminated the increased viability of HAECs induced by miR-34a inhibitor (Fig. 5). These results indicated that miR-34a inhibitor enhances the cell viability of HAECs through regulating HDAC1.

MiR-34a inhibitor suppresses apoptosis in HAECs. Flow cytometry analysis demonstrated that cell apoptosis were increased in Ox-LDL induction HAECs compared to the control group. Compared with ox-LDL treatment alone, miR-34a inhibitor significantly reduced the apoptosis of HAECs, whereas HDAC1-siRNA eliminated the decrease of apoptosis of HAECs induced by miR-34a inhibitor (Fig. 6). Together, these data indicated that miR-34a inhibits cell grow and promotes apoptosis of HAECs via regulating HDAC1.

Effects of miR-34a inhibitor on the expression of apoptosis-related genes. To further investigate the molecular mechanism of the miR-34a effects on HAECs, the expression of apoptosis-related genes were detected by western blotting.

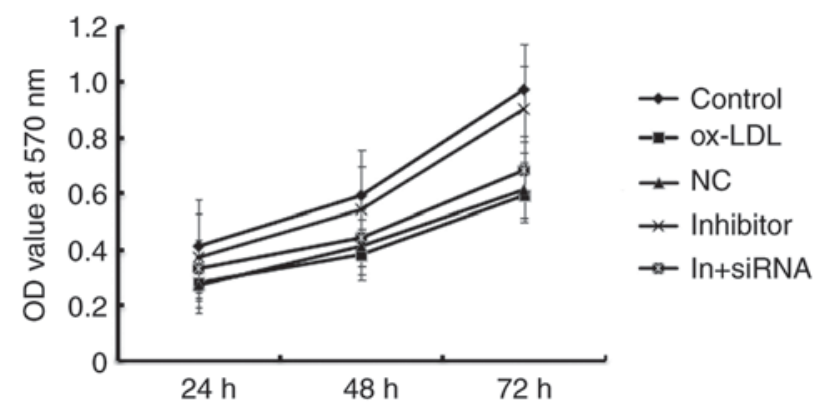

Figure 5. Effect of miR-34a inhibitor on the cell viability of HEACs. $48 \mathrm{~h}$ after cell transfection, CCK-8 assay was used to detect the cell viability of HEACs. All data are presented as the mean \pm SD of three independent experiments.

The results showed that miR-34a inhibitor significantly increased the expression of Bcl-2, procaspase-3, procaspase-9 and c-Myc, and the expression of p21 was significantly decreased compared with ox-LDL treatment alone. In addition, HDAC1-siRNA eliminated the effects induced by miR-34a inhibitor (Fig. 7). It is further demonstrated that miR-34a regulates the expression of apoptosis-related proteins by targeting HDAC1.

\section{Discussion}

Functional miRNA of miR-34a have been highly concerned by researchers in recent years. Welch et al (13), reported that ectopic miR-34a induces apoptosis resulting in the activation of a caspase-mediated apoptotic pathway when reintroduced into the neuroblastoma cell lines, which show a decrease in expression of miR-34a. Then, a number of data showed that miR-34a regulates a plethora of target proteins to induce cell 


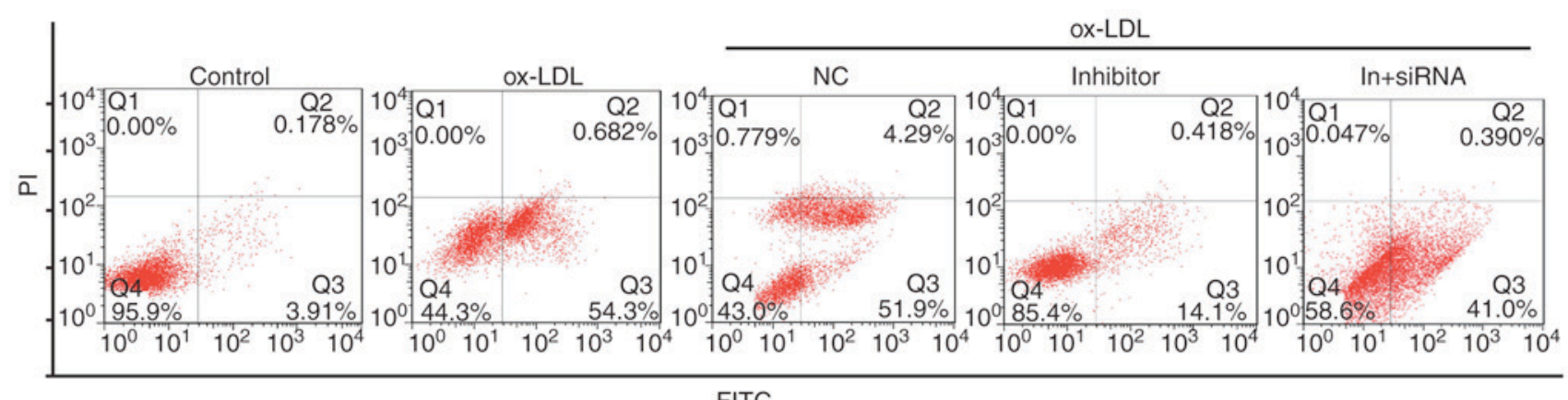

FITC

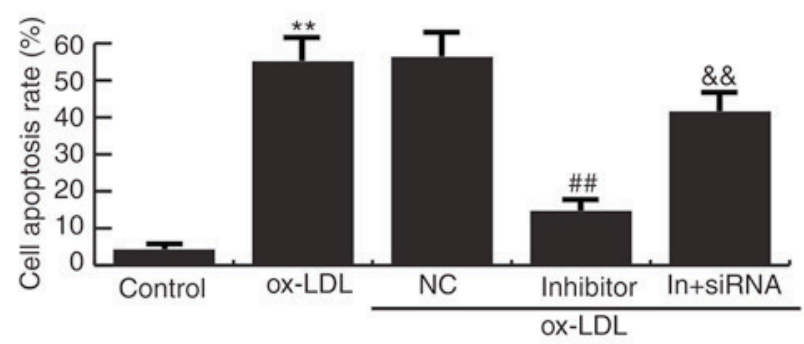

Figure 6. Effect of miR-34a inhibitor on the cell apoptosis of HEACs. $48 \mathrm{~h}$ after cell transfection, FCM was used to detect the cell apoptosis of HEACs, and data was analyzed. All data are presented as the mean $\pm \mathrm{SD}$ of three independent experiments. ${ }^{* *} \mathrm{P}<0.01 \mathrm{vs}$. control; ${ }^{\# \#} \mathrm{P}<0.01 \mathrm{vs}$. oxLDL; ${ }^{\&} \mathrm{P}<0.01 \mathrm{vs}$. inhibitor.
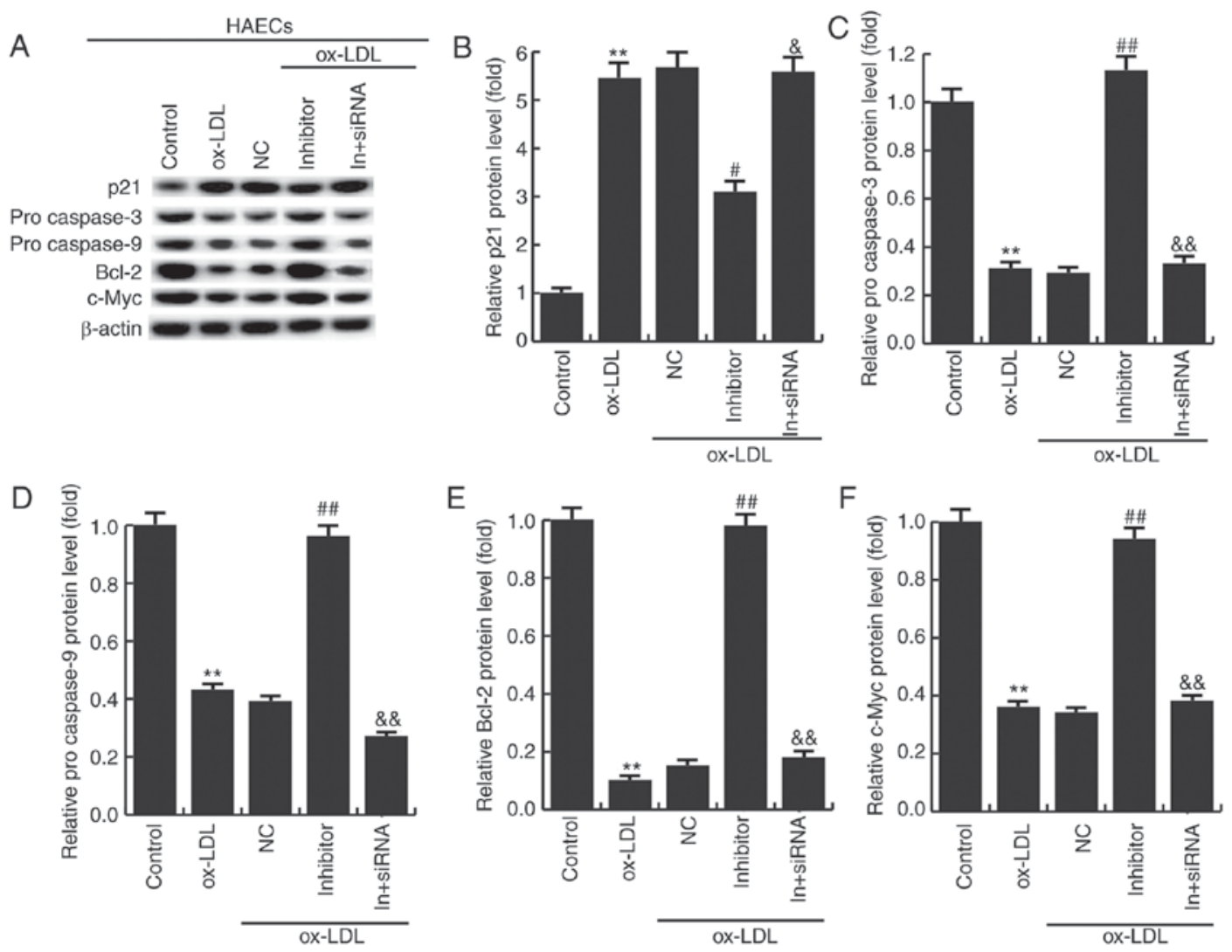

Figure 7. Effect of miR-34a inhibitor on cell apoptosis related proteins expression in HEACs. (A) $48 \mathrm{~h}$ after cell transfection, western blotting was used to detect the protein expression of Bcl-2, procaspase-3, procaspase-9, c-Myc and p21, and (B-F) protein expression was quantified and presented as fold of the control. All data are presented as the mean $\pm \mathrm{SD}$ of three independent experiments. ${ }^{* *} \mathrm{P}<0.01 \mathrm{vs}$. control; ${ }^{*} \mathrm{P}<0.05,{ }^{\# \#} \mathrm{P}<0.01 \mathrm{vs}$. oxLDL; ${ }^{\&} \mathrm{P}<0.05$, ${ }^{\text {\& }} \mathrm{P}<0.01 \mathrm{vs}$. inhibitor.

apoptosis, thus acting as a tumor suppressor (14-17). The present study found that miR-34a inhibitor could repress cell apoptosis in atherosclerosis.

In the present study, our preliminary data showed that miR-34a was significantly increased in atherosclerotic lesion tissues compared with the normal tissues, and consistent results was found in ox-LDL induced HAECs. Also, we found that the expression of HDAC1 was negative correlation with miR-34a. So we hypothesis HDAC1 gene was associated with miR-34a. In order to confirm this hypothesis, firstly we use 
TargetScan to predict the potential targets of miR-34a. HDAC1 was identified as a potential miR-34a target gene. In addition, luciferase reporter assay verified miR-34a directly binding to 3'-UTR of HDAC1. Moreover, the expression of HDAC1 was significantly increased in miR-34a inhibitor transfected HAECs. These results demonstrated that miR-34a directly targets HDAC1.

Histone acetylation function as reducing histone-DNA interactions or creating an open chromatin configuration (18). Histone deacetylases (HDAC)-dependent regulation of protein acetylation levels leads to cell- and gene-specific transcriptional repression or activation (18). HDAC1 is considered as a positive regulator of cell proliferation, HDAC1 depletion in mice results in growth deficiencies, and the p21 cyclin-dependent inhibitor were correlated increased $(19,20)$. Our research have revealed that miR-34a directly targets HDAC1, and miR-34a inhibitor could promote cell viability and prevent cell apoptosis. We further explored underlying mechanism of the effects on HAECs caused by miR-34a, and the relationship between HDAC1 and apoptosis was explored. The expression levels of the anti-apoptotic protein Bcl-2, procaspase-3, procaspase- 9 and c-Myc were detected after transfection with miR-34a inhibitor or miR-34a inhibitor +HDAC1-siRNA. Results showed that miR-34a inhibitor significantly improved the expression of anti-apoptotic protein, and HDAC1-siRNA eliminated the effects of miR-34a inhibitor on HAECs.

Moreover, p21 was also determined in the present study, and the findings suggested that miR-34a inhibitor significantly inhibited p21 expression, and this inhibition was reversed by HDAC1-siRNA. It has been demonstrated that the most significant down-regulation by miR-34a is represented by ribosomal proteins (17). Of interest, some ribosomal proteins have the ability to up-regulate miR-34 and down-regulate p21. Established anticancer treatments were recently introduced into atherosclerosis therapeutic strategies to prevent restenosis after angioplasty and endarterectomy. miR-34a increases the sensitivity of cells to 5-FU treatment (21) and recently it has been demonstrated that some ribosomal proteins are essential to mediate cell apoptotis caused by 5-FU through molecular mechanisms involving crucial pro-inflammatory factors as NFkB (22). In the light of these findings, one possibility is that miR-34a inhibitor down-regulated p21 expression through regulating ribosomal proteins thus preventing cell apoptosis.

In conclusion, this study demonstrated that miR-34a was up-regulated in atherosclerosis. MiR-34a inhibition could promote the proliferation and induce apoptosis of endothelial cells through regulating the expression of apoptotic-associated proteins by targeting HDAC1. Thus, our research provides evidence that miR-34a might serve as a new potential therapeutic target for atherosclerosis.

\section{References}

1. Libby P and Theroux P: Pathophysiology of coronary artery disease. Circulation 111: 3481-3488, 2005.

2. Williams KJ and Tabas I: Atherosclerosis and inflammation. Science 297: 521-522, 2002 .

3. Hansson GK and Libby P: The immune response in atherosclerosis: A double-edged sword. Nat Rev Immunol 6: 508-519, 2006.

4. Hansson GK and Hermansson A: The immune system in atherosclerosis. Nat Immunol 12: 204-212, 2011.

5. Hammond SM: An overview of microRNAs. Adv Drug Deliv Rev 87: 3-14, 2015.

6. Wilson RC and Doudna JA: Molecular mechanisms of RNA interference. Annu Rev Biophys 42: 217-239, 2013.

7. Guo H, Ingolia NT, Weissman JS and Bartel DP: Mammalian microRNAs predominantly act to decrease target mRNA levels. Nature 466: 835-840, 2010.

8. Miska EA: How microRNAs control cell division, differentiation and death. Curr Opin Genet Dev 15: 563-568, 2005.

9. Hermeking H: The miR-34 family in cancer and apoptosis. Cell Death Differ 17: 193-199, 2010

10. Ito T, Yagi S and Yamakuchi M: MicroRNA-34a regulation of endothelial senescence. Biochem Biophys Res Commun 398: 735-740, 2010.

11. Zhao T, Li J and Chen AF: MicroRNA-34a induces endothelial progenitor cell senescence and impedes its angiogenesis via suppressing silent information regulator 1. Am J Physiol Endocrinol Metab 299: E110-E116, 2010.

12. Livak KJ and Schmittgen TD: Analysis of relative gene expression data using real-time quantitative PCR and the 2(-Delta Delta C(T)) method. Methods 25: 402-408, 2001.

13. Welch C, Chen Y and Stallings RL: MicroRNA-34a functions as a potential tumor suppressor by inducing apoptosis in neuroblastoma cells. Oncogene 26: 5017-5022, 2007.

14. Cole KA, Attiyeh EF, Mosse YP, Laquaglia MJ, Diskin SJ, Brodeur GM and Maris JM: A functional screen identifies miR-34a as a candidate neuroblastoma tumor suppressor gene. Mol Cancer Res 6: 735-742, 2008.

15. Fujita Y, Kojima K, Hamada N, Ohhashi R, Akao Y, Nozawa Y, Deguchi $\mathrm{T}$ and Ito M: Effects of miR-34a on cell growth and chemoresistance in prostate cancer PC3 cells. Biochem Biophys Res Commun 377: 114-119, 2008.

16. Ji Q, Hao X, Meng Y, Zhang M, Desano J, Fan D and Xu L: Restoration of tumor suppressor miR-34 inhibits human p53-mutant gastric cancer tumorspheres. BMC Cancer 8: 266, 2008.

17. Chen QR, Yu LR, Tsang P, Wei JS, Song YK, Cheuk A, Chung JY, Hewitt SM, Veenstra TD and Khan J: Systematic proteome analysis identifies transcription factor YY1 as a direct target of miR-34a. J Proteome Res 10: 479-487, 2011.

18. Reichert N, Choukrallah MA and Matthias P: Multiple roles of class I HDACs in proliferation, differentiation and development. Cell Mol Life Sci 69: 2173-2187, 2012.

19. Jurkin J, Zupkovitz G, Lagger S, Grausenburger R, Hagelkruys A, Kenner $\mathrm{L}$ and Seiser C: Distinct and redundant functions of histone deacetylases HDAC1 and HDAC2 in proliferation and tumorigenesis. Cell Cycle 10: 406-412, 2011.

20. Haberland M, Montgomery RL and Olson EN: The many roles of histone deacetylases in development and physiology: Implications for disease and therapy. Nat Rev Genet 10: 32-42, 2009.

21. Li X, Zhao H, Zhou X and Song L: Inhibition of lactate dehydrogenase A by microRNA-34a resensitizes colon cancer cells to 5-fluorouracil. Mol Med Rep 11: 577-582, 2015.

22. Russo A, Saide A, Cagliani R, Cantile M, Botti G and Russo G: rpL3 promotes the apoptosis of p53 mutated lung cancer cells by down-regulating CBS and NFKB upon 5-FU treatment. Sci Rep 6: 38369, 2016. 